\title{
Thermophysical Property and Pore Structure Evolution in Stressed and Non- Stressed IG-110 Nuclear Graphite
}

\author{
L. L. Snead ${ }^{1, *}$, C. I. Contescu, ${ }^{2,}$, T. S. Byun ${ }^{3}$, and W. Porter ${ }^{2}$ \\ 1,* Massachusetts Institute of Technology, Cambridge MA \\ 2 Oak Ridge National Laboratory, Oak Ridge TN \\ ${ }^{3}$ Pacific Northwest National Laboratories, Richland WA \\ *corresponding author
}

\begin{abstract}
The nuclear graphite, IG-110, was irradiated with and without a compressive load of 5 $\mathrm{MPa}$ at $\sim 400^{\circ} \mathrm{C}$ up to $9.3 \times 10^{25} \mathrm{n} / \mathrm{m}^{2}(\mathrm{E}>0.1 \mathrm{MeV}$.) Following irradiation physical properties were studied to compare the effect of graphite irradiation on microstructure developed under compression and in stress-free condition. Properties included: dimensional change, thermal conductivity, dynamic modulus, and CTE. The effect of stress on open internal porosity was determined through nitrogen adsorption. The IG-110 graphite experienced irradiation-induced creep that is differentiated from irradiationinduced swelling. Irradiation under stress resulted in somewhat greater thermal conductivity and coefficient of thermal expansion. While a significant increase in dynamic modulus occurs, no differentiation between materials irradiated with and without compressive stress was observed. Nitrogen adsorption analysis suggests a difference in pore evolution in the $0.3-40 \mathrm{~nm}$ range for graphite irradiated with and without stress, but this evolution is seen to be a small contributor to the overall dimensional change.
\end{abstract}




\section{Introduction}

Graphite is an important material for a number of reactor systems and particularly for high temperature gas cooled reactors for which it comprises the bulk of the core material. Under neutron irradiation, the graphite microstructure is known to undergo gross microstructural evolution resulting in similarly large property changes. [1] One area of historic and continuing research is that of irradiation creep of nuclear graphite. This irradiation creep, defined as the difference between the irradiation-induced growth of graphite in a stress-free condition and the irradiation-growth under a stressed condition, is of great technical importance to reactor design. As is well understood [1], without the stress relief provided by irradiation creep, failure due to the elastic strain caused by irradiation-induced swelling of graphite would preclude its use. Irradiation creep (an inelastic strain) occurs over essentially all temperatures and independently of thermal creep and has been modeled extensively with varying degrees of success. [2] The purpose of this paper is to firstly provide a simple technique for carrying out graphite creep experiment, and secondly demonstrate how that technique can be used to generate key information on physical properties and microstructural evolution of graphite under load.

\section{Experimental}

\subsection{Materials, Irradiation, and Irradiation Vehicle}

The material chosen for this study was the nuclear grade IG-110 graphite manufactured by Toyo Tanso. This graphite is derived from petroleum coke and fabricated through cold isotropic pressing (isomolding). A typical optical micrograph of a polished section of this material is given in Figure 1. This superfine nuclear graphite is composed of needle-shaped filler particle with a manufacturer specification of approximately 20 microns. This information was confirmed by a recent optical microscopy study of IG-110 graphite with the same pedigree as those studied here [3] which determined that average particle length is $27 \pm 2 \mu \mathrm{m}$ and the average aspect ratio is $3.9 \pm 0.2$. It is noted that the manufacturer quoted grain size is $10 \mu \mathrm{m}$. Occasionally, much larger needle shaped particles can be found. An example of the less common, larger particles surrounded by the more common microstructure is provided in Fig. 1 for a slow-cure epoxy potted samples of non-irradiated IG-110. The overall porosity for IG-110 is $\sim 18 \%$. As compared to extruded forms of nuclear graphite, the majority of pore volume for isomolded IG-110 is associated with pore sizes (diameter of effective cylinder) in the range of 0.005 to $0.5 \mu \mathrm{m}$. [4] As example, Gilsocarbon(1 or 2) and SGL Carbon NBG18 nuclear graphite have significant volumes associated with porosity in the 10-150 $\mu \mathrm{m}$ range. 
$\mathrm{X}$-ray analysis indicates that the graphite crystallites that compose filler grains have much larger average size in $\langle a\rangle$ direction $\left(L_{a}=256 \AA\right)$ than in $\langle c\rangle$ direction $\left(L_{c}=190 \AA\right)$. [5] Since the major axis of needle-shaped particles is parallel with $\langle a>$ direction of graphite crystallites, the shrinkage cracks that run along these long particles are bounded by graphite basal planes. In fact, a calculation based on XRD crystallite parameters and using a model for natural graphite flakes [6] indicates that basal planes compose about 73 $\%$ of the exposed surface of graphite grains in IG-110. [4]

While this graphite is described as isotropic, important thermophysical properties such as electrical conductivity, elastic modulus, and coefficient of thermal expansion (CTE, $\alpha$ ) have a slight (few percent) variation in the direction parallel to the gravity axis during forming (with gravity: WG) and perpendicular to the gravity vector (against gravity: AG.) Moreover, this minor anisotropy is known to carry over and perhaps magnify in the as-irradiated properties.[7] For this work IG-110 cylinders were machined with the cylinder axis in the WG direction only.

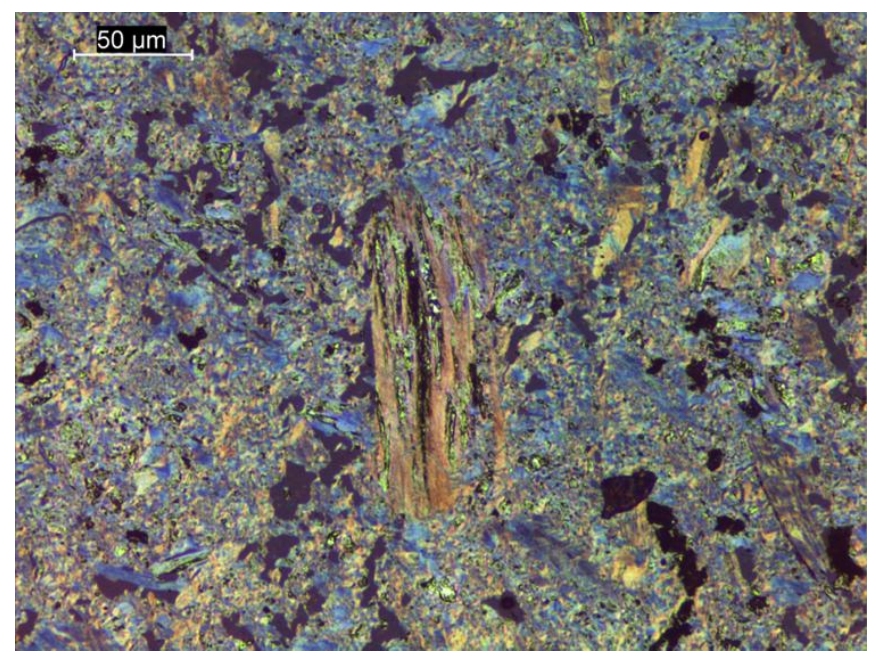

Figure 1: Polarized light micrograph of non-irradiated IG-110 graphite.

A new technique was developed to apply in-reactor load to these graphite specimens. Specifically, a compact internally pre-pressurized bellows load frame was developed and is described in detail elsewhere. [8] Briefly, the cylindrical graphite specimen and the bellows are placed within a load frame. This load frame is then fitted inside the irradiation housing that externally is in contact with the reactor coolant. The graphite specimen within the load frame is precisely machined to $6 \mathrm{~mm}$ in diameter and includes a $1 \mathrm{~mm}$ axi-symmetric through-hole into which a $\mathrm{SiC}$ temperature monitor is placed. Once the bellows and load frame are secured within the holder the bellows is pressurized with helium through an end-hole in the bellows. Upon achieving desired pressure (thus desired contact pressure and stress on graphite sample) the hole is sealed by laser welding. A pressure equivalent to approximately $5 \mathrm{MPa}$, predicted for the enclosure at the irradiation temperature, was chosen. It is noted that the pressure at temperature was not measured prior to irradiation, but was measured at room temperature and extrapolated assuming ideal gas law increase. It is possible, as measured in subsequent bellows- 
loaded irradiated vehicles, that some level of pressure loss occurs during irradiation. A photo of the irradiation vehicle before and after load-frame assembly is provided in Figure 2.

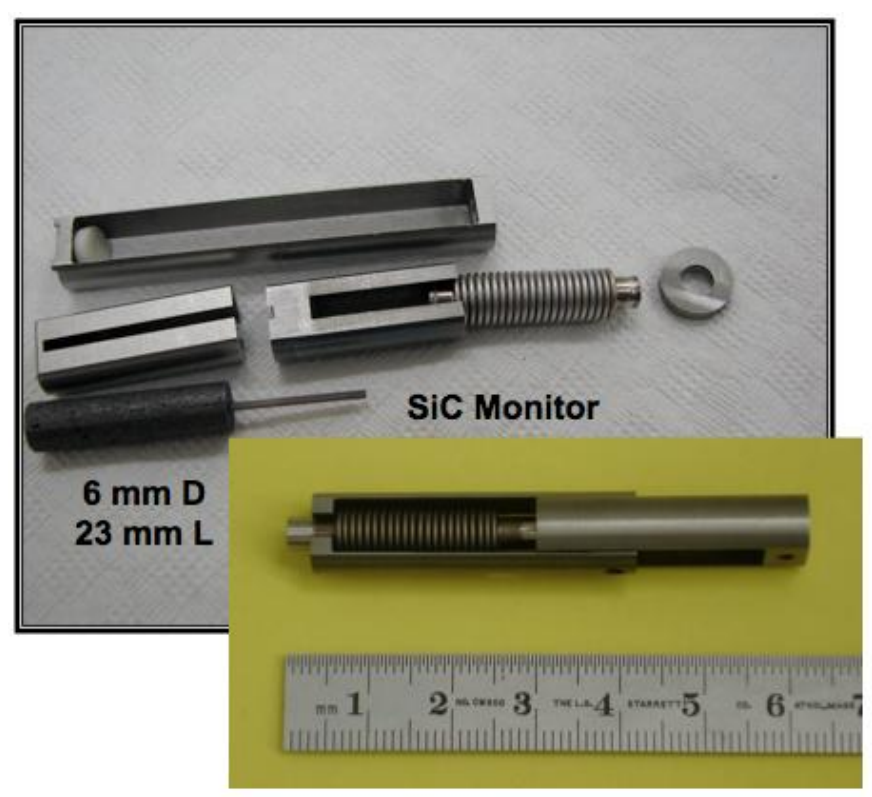

Figure 2: Components of graphite creep capsule. A detailed description of the irradiation vehicle including applied stress is provided elsewhere. [8]

As irradiation-induced creep in graphite occurs in concert with appreciable irradiationinduced dimensional change, a "matched-pair" approached is used to elucidate the creep component. Samples were irradiated in as near identical conditions as possible: for each loaded capsule an identical non-pressurized free capsule was irradiated in a mirrored flux position within the reactor core. This provided a side-by-side comparison of loaded and free samples allowing the effect of irradiation in the absence of load to be accounted for. The irradiation was carried out in the High Flux Isotope Reactor (HFIR) of the Oak Ridge National Laboratory in the central flux trap position, thereby accumulating the greatest rate of fast neutron damage. A total of eight capsules, referred to as the R11-A1 through A8, were irradiated in the TRRH-4 position providing a fast neutron flux $(\mathrm{E}>0.1$ $\mathrm{MeV}$ ) of $1.09 \times 10^{19} \mathrm{n} / \mathrm{m}^{2}$-s. The capsules were irradiated in four matched-pairs for one, two, three, and four HFIR cycles.

Irradiation temperature in this vehicle was achieved through a balance of internal heat generation due to the gamma heating of the vehicle component and the conduction of that heat across a defined gas-gap between the load-frame and the aluminum housing. The target temperature was achieved by selection of capsule fill gas and setting the conduction gap. As the cylindrical sample is in hard-contact at the end faces of the sample axial conduction is a significant contributor to the sample heat transfer. Three dimensional heat transfer analysis was used to determine the axial temperature gradient. In the thermal analysis the heat input to the capsule was taken as constant, a typical assumption given the steady power profile for HFIR, as was the gas-gap between the 
external housing and load frame. Time and spacial dependence of sample temperature was due to the radial and axial conduction from the sample to the holder and bellows-end anvil, respectively. The effect of graphite sample shrinkage would be to increase the radial gap between sample and holder, thereby decreasing conduction away from sample. The effect of irradiation-induced reduction in sample thermal conductivity would be to increase the thermal resistance to the bellows-anvil. As will be seen in the results section the sample shrinkage continues throughout the experiment while thermal conductivity saturates at the lower fluences of this experiment. The result of these changes induces as much as a $40^{\circ} \mathrm{C}$ axial gradient along the highest fluence sample, with the coolest end being that in contact with the bellows-anvil.

The $\mathrm{SiC}$ temperature monitors were extracted, cleaned, and processed using the CTE method to determine irradiation temperature. Such temperature monitors are now in routine use, providing accurate information on the irradiation temperature near the end of irradiation. [9] [10] Figure 3 provides the differential length change as a function of temperature for a constant measurement temperature ramp rate of $2.5 \mathrm{~K} /$ minute in argon. The difference refers to the difference between the increasing length due to thermal expansion and the decreasing length that occurs due to annealing of point defect cluster when the annealing temperature exceeds the irradiation temperature. A recent publication by Campbell discussed the use of differential length change for determination of post-irradiation temperature monitoring. [11] In this process, when the measurement temperature exceeds the irradiation temperature the irradiation-created defects that were stable up to the irradiation temperature begin to annihilate causing the sample to shrink. The point of shrinkage is defined as the irradiation temperatures, and is indicated by the dashed lines in Figure 3. Recalling the axial temperature profile of the graphite specimen, the temperature monitor mirrors this profile, with the point of shrinkage in Figure 3 occurring at the lowest irradiation temperature along the monitor. Unfortunately, the temperature monitor for the capsule R11-A8 did not survive disassembly and we have no measured confirmation of temperature for that capsule. However, noting the difference in measured irradiation temperatures for each of the matched samples (A1/A2, A3/A4, and A5/A6) it can be inferred that the irradiation temperature for R1l-A 8 should be within $\sim 20^{\circ} \mathrm{C}$ of the R11-A7 capsule (i.e. $421 \pm 20^{\circ} \mathrm{C}$.)

Following irradiation, prior to extraction of the graphite sample, the integrity of the loadframe was determined by compression testing as described elsewhere [8]. Bellows loading was carried out at the measured irradiation temperature with load increasing until the point of bellows compression. In simple terms, the stress applied to the sample is determined from the point at which the applied load to the bellows indicates bellows displacement (applied force overcoming bellows-force). Unfortunately, while the matched-pair bellows for the first two cycles yielded smooth frame movement and expected contact pressures, the two higher fluence mated-pairs were problematic. In both cases the load-frame did not smoothly yield upon loading (showing a degree of binding.) Therefore the point of bellows displacement could not be easily determined and therefore the end of irradiation stress is unknown. Moreover, for two upper fluences, once the bellow did move within the frame the movement was not smooth, indicating some level of load train binding. Table 1 provides information specific to the irradiation capsules, 
including the assumed applied pressure for the two lower fluence capsules. No information with regard to the upper fluence capsules is provided (listed as "unmeasurable.") It is noted that the stress applied to the samples was not measured prior to reactor as described above, though the results of the two low-fluence capsules agree well with expectations given the initial fill gas. In any event, as pre-irradiation stress was not measured the data generated here should only be used for design purposes with caution.

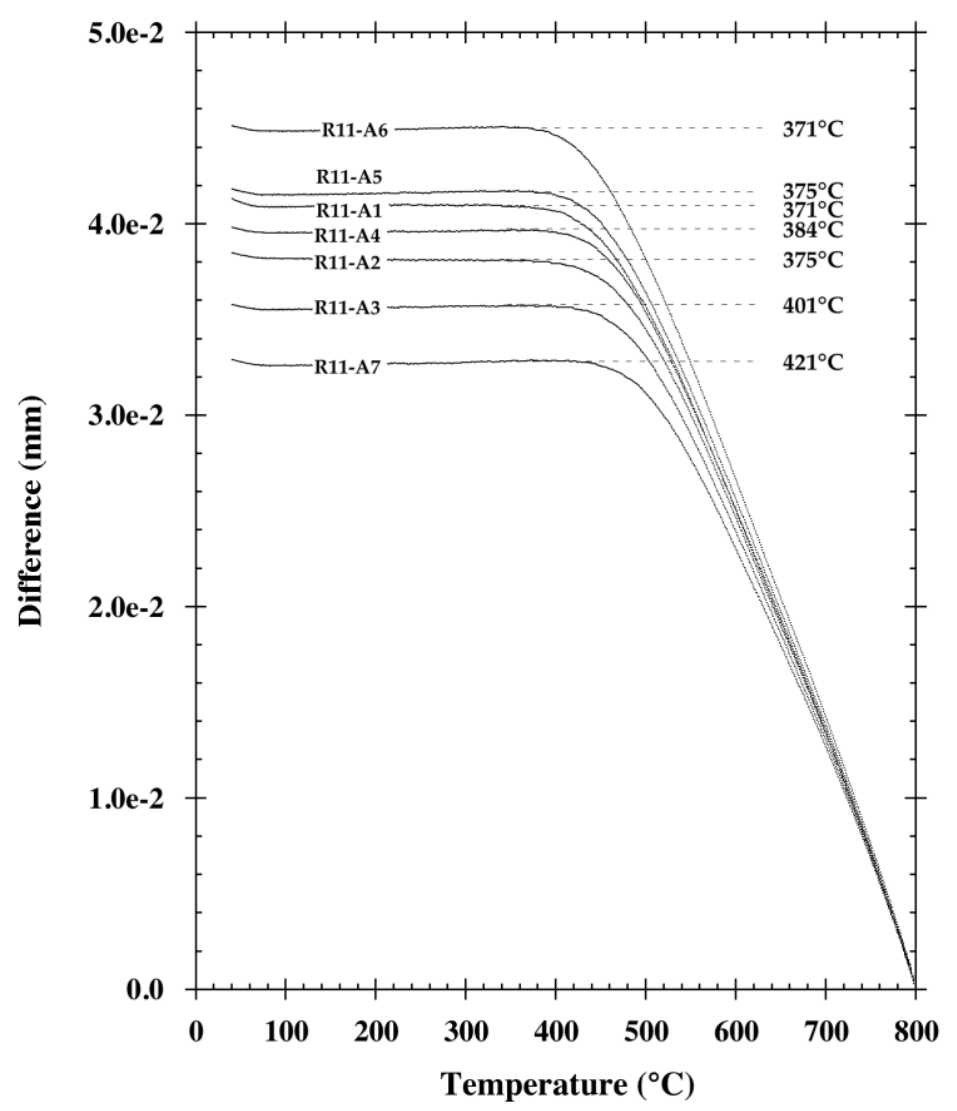

Figure 3: Irradiation temperature determination through post-irradiation isochronal anneal and CTE measurement.

\begin{tabular}{|c|c|c|c|c|}
\hline & $\begin{array}{c}\text { Fluence } \\
\left(\mathrm{n} / \mathrm{m}^{2}\right) \\
\mathrm{E}>0.1 \mathrm{MeV} \\
\mathrm{x} 10^{25}\end{array}$ & $\mathrm{dpa}^{*}$ & $\begin{array}{c}\text { Irradiation } \\
\text { Temperature }\left({ }^{\circ} \mathrm{C}\right)\end{array}$ & $\begin{array}{c}\text { Applied Stress at } 400^{\circ} \mathrm{C} \\
\text { At End of Irradiation } \\
(\mathrm{MPa})\end{array}$ \\
\hline R11-A1 & 2.4 & 1.75 & 371 & 4.9 \\
\hline $\mathrm{R} 11-\mathrm{A} 2$ & 2.4 & 1.75 & 375 & 0 \\
\hline $\mathrm{R} 11-\mathrm{A} 3$ & 4.6 & 3.36 & 401 & 5.4 \\
\hline R11-A4 & 4.6 & 3.36 & 384 & 0 \\
\hline R11-A5 & 7.1 & 5.12 & 375 & Un-measurable \\
\hline R11-A6 & 7.1 & 5.12 & 371 & 0 \\
\hline
\end{tabular}




\begin{tabular}{|c|c|c|c|c|}
\hline R11-A7 & 9.3 & 6.79 & 421 & Un-measurable \\
\hline R11-A8 & 9.3 & 6.79 & - & 0 \\
\hline
\end{tabular}

* Conversion of $1 \mathrm{dpa}=0.73 \times 10^{25} \mathrm{E}>0.1 \mathrm{MeV}$

Table 1: R11 Series Capsule Information.

Regardless of this internal binding the bellows would still be able to apply compressive load to graphite specimen to a certain point through a deformation in free shoulder of bellows. The width of free shoulder is about $1.5 \mathrm{~mm}$ in the bellows [9], and with internal pressure the free shoulder can deform in the axial direction to conform to the contraction in specimen. Such axial direction expansion should be effective until the axial displacement at specimen contact reaches its limit. It is expected to be only a fraction of $\mathrm{mm}$ or a few percent strain in the graphite specimen even with irradiation enhanced creep. Before the limit displacement is reached, therefore, the irradiation creep data with binding can be considered to be valid.

\subsection{Thermophysical Property Measurements}

Thermal diffusivity was measured using a Netzsch LFA 457 MicroFlash Laser Flash thermal diffusivity apparatus. Samples of approximately $3 \mathrm{~mm}$ in thickness were sectioned from the cylindrical sample following irradiation and diffusivity as a function of temperature measured. Thermal conductivity was calculated following the standard formalism: $\mathrm{K}=\alpha \rho \mathrm{C}_{\mathrm{p}}$ where $\alpha$ is the thermal diffusivity found from the measured Cowan half-rise time, $\rho$ is the density measured by mass and physical dimension and $C_{p}$ is the temperature-dependent specific heat. The guiding standard utilized was ASTM E-1461 Standard Test Method for Thermal Diffusivity by the Flash Method. Coefficient of thermal expansion for graphite specimens and temperature monitors were measure asirradiated, intact, using a Netzsch DIL 402CD Thermal Expansion Dilatometer. For the graphite specimens the specimen expansion was measured over a temperature range from room temperature to just under the measured irradiation temperature at a temperature ramp-rate of $3^{\circ} \mathrm{K} /$ minute. The elastic modulus was measured on intact specimens using a J. W. Lemmens GrindoSonic Mk5 following the ASTM C747 Standard Test Method for Moduli of Elasticity and Fundamental Frequencies of Carbon and Graphite Materials by Sonic Resonance. For the non-contact measurement of the sample dimensions a BETA LaserMike Model 162 Scanning Optical Micrometer and associated dimensional standards were used. In all cases where physical dimensions and mass were measured calibrated instruments were utilized.

\subsection{Nitrogen Porosimetry}

Nitrogen adsorption at $77 \mathrm{~K}$ was used to characterize the effect of neutron irradiation on the internal surface and nitrogen accessible porosity of IG-110 graphite. Measurements were carried out by the volumetric method using the Autosorb 1 instrument (Quantachrome Instruments) on virgin and irradiated specimens. Before measurements all specimens were outgassed overnight at $300{ }^{\circ} \mathrm{C}$ in vacuum $(<0.2 \mathrm{~Pa})$. Adsorption data 
were processed by the standard procedures used for calculation of the specific surface area by the Brunauer - Emmett - Teller (BET) method [12] , the total volume of open pores, and the average pore size.

\section{Results and Discussion}

\subsection{Dimensional Change in the Loaded and Free Condition}

The dimensional change for the free and loaded IG-110 graphite specimens along the length of the cylindrical sample is provided in Figure 4, with the change in diameter provided in Figure 5. In comparing fig 5 with fig 4 the error bars associated with the diameter change ( \pm one standard deviation translated to percent difference) are more pronounced for the diameter due to both the smaller dimension and non-uniformity of the disc specimens. It is noted that the laser micrometer was utilized for the measurement of diameter while standard micrometers were used for length. From Figure 4 a monotonic length decrease is apparent for the samples free of external loading. Unfortunately, a good data-set for comparison does not exist for dimensional change of IG-110 or similar graphite irradiated near $400^{\circ} \mathrm{C}$, [13] though limited data exists in the temperature range of $600-1000^{\circ} \mathrm{C}$. Kunimoto and co-workers [14] have conducted an analysis extrapolating the irradiation effects of a number of graphite types in order to predict IG-110 thermophysical behavior as a function of irradiation temperature and fluence. Following Kunimoto's analysis for the irradiation conditions of the current study, IG-110 is predicted to undergo monotonic shrinkage, though at the lowest fluence a greater shrinkage is predicted (about $-0.45 \%$ as compared to the measured $-0.25 \%$.) However, their prediction agrees well with the free dimensional change in Figure 4 for the upper fluences. Under compressive loading the IG-110 samples clearly exhibits irradiation creep behavior, defined as the difference in length change between the loaded and free samples in Figure 4. As discussed in the experimental section, the capsule associated with the loaded $7.1 \times 10^{25}$ (E>0.1 MeV) fluence indicated load-train binding. However, given that the length change for the loaded sample continues to increase for the $7.1 \times 10^{25}$ $(\mathrm{E}>0.1 \mathrm{MeV})$ it is speculated that any issue with the applied loading occurred during the third irradiation cycle, or after the lower irradiation dose of Figure $4\left(4.6 \times 10^{25}(\mathrm{E}>0.1\right.$ $\mathrm{MeV}$ ).) In contrast, by inspection of the four-cycle dimensional change of Figure 4 the load train has apparently retained load through the complete irradiation period, though this cannot be confirmed. 


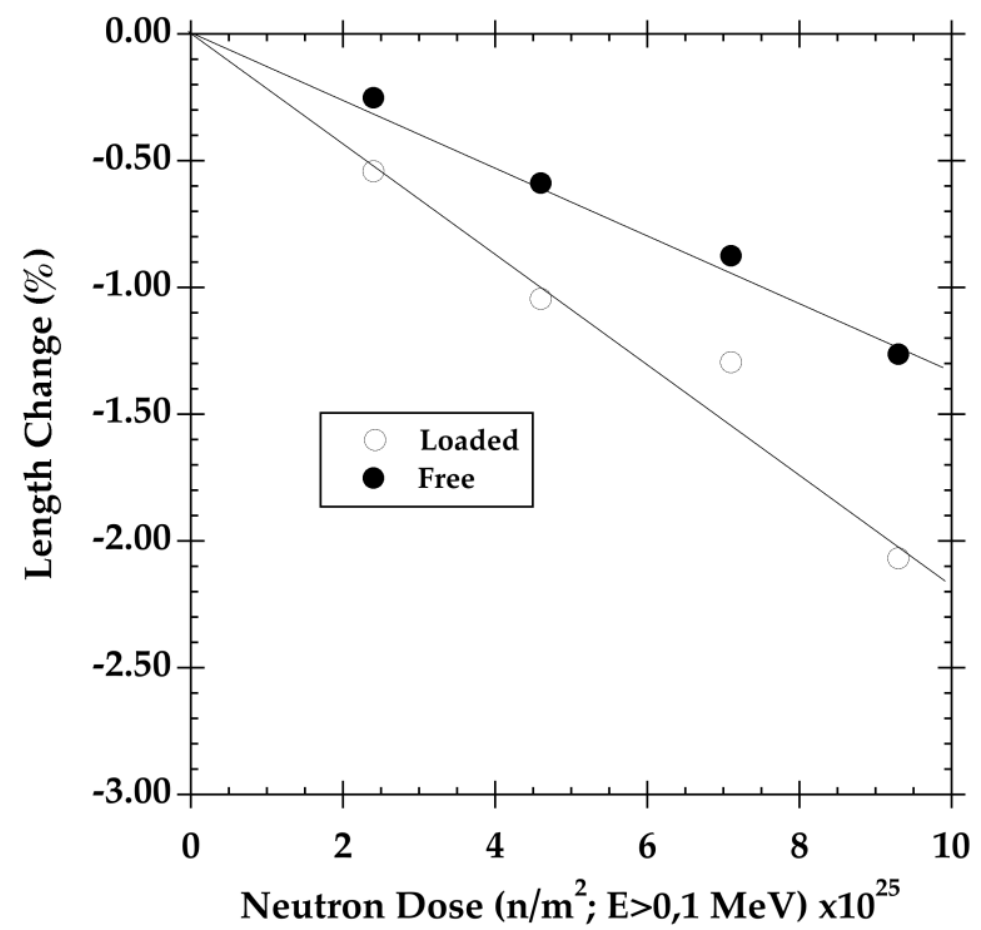

Figure 4: Length change for the loaded and free IG-110 specimen as a function of neutron fluence. Irradiation temperature $371-421^{\circ} \mathrm{C}$.

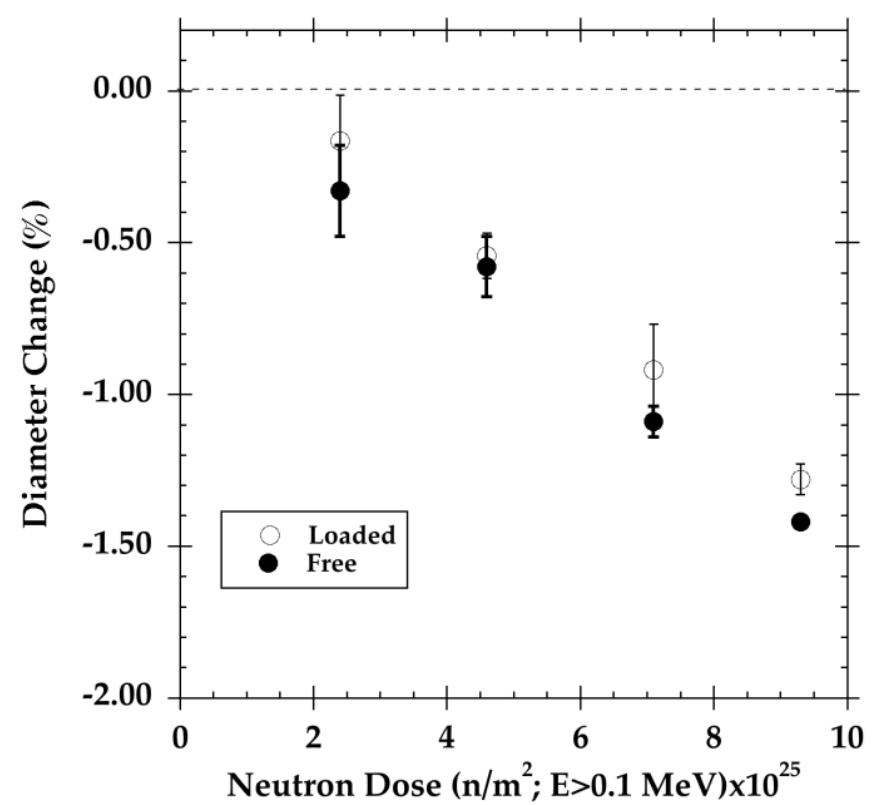

Figure 5: Diameter change for the loaded and free IG-110 specimen as a function of neutron fluence. Irradiation temperature $371-421^{\circ} \mathrm{C}$.

The diameter change data provided in Figure 5 is determined by using a laser profilometer. From the figure the absolute difference in shrinkage between loaded and free sample is relatively minimal with the impact of the compressive load being restraint 
of diameter shrinkage. The companion figure (Figure 6) addresses a design concern regarding the bellows-loaded sample. Specifically, the possibility of non-uniform or nonaxial stress due to imperfect coupling between the bellows loading anvil and the sample may result in bulging or non-uniform diameter change near the loaded end of the sample. Figure 6 provides the diameter change as a function of length for the loaded and free sample for the highest fluence level (R11-A7, $9.3 \times 10^{25} \mathrm{n} / \mathrm{m}^{2}$ ) as provided by the laser profilometer. Three points can be made through inspection of the data of this graph. First, a small negative diameter change $(-1.28 \%)$ has occurred due to irradiation (accounting for $<10 \%$ total increase in gas gap). The second observation from Figure 6 is a minor change in surface uniformity along the length has occurred. The laser profilometry data, taken in a spiral pattern along the length of the cylinder, has clearly indicated a lack of bulging near the loaded or restrained ends of the sample and nearsymmetry in the dimensional change, supporting the validity of the bellows load train for carrying out creep testing. The third observation that can be made from Figure 6, which was consistent with all paired samples, is that the as-irradiated surface was slightly rougher following irradiation. No conclusion is made with regard to the mechanism for this, though appears to be an artifact of irradiation, consistent with surface morphological change occurring due to anisotropic crystallite change, as it occurred in both loaded and free samples. However, some roughening due to oxidation cannot be ruled out, though care was taken to utilize high-purity helium as the capsule fill gas.

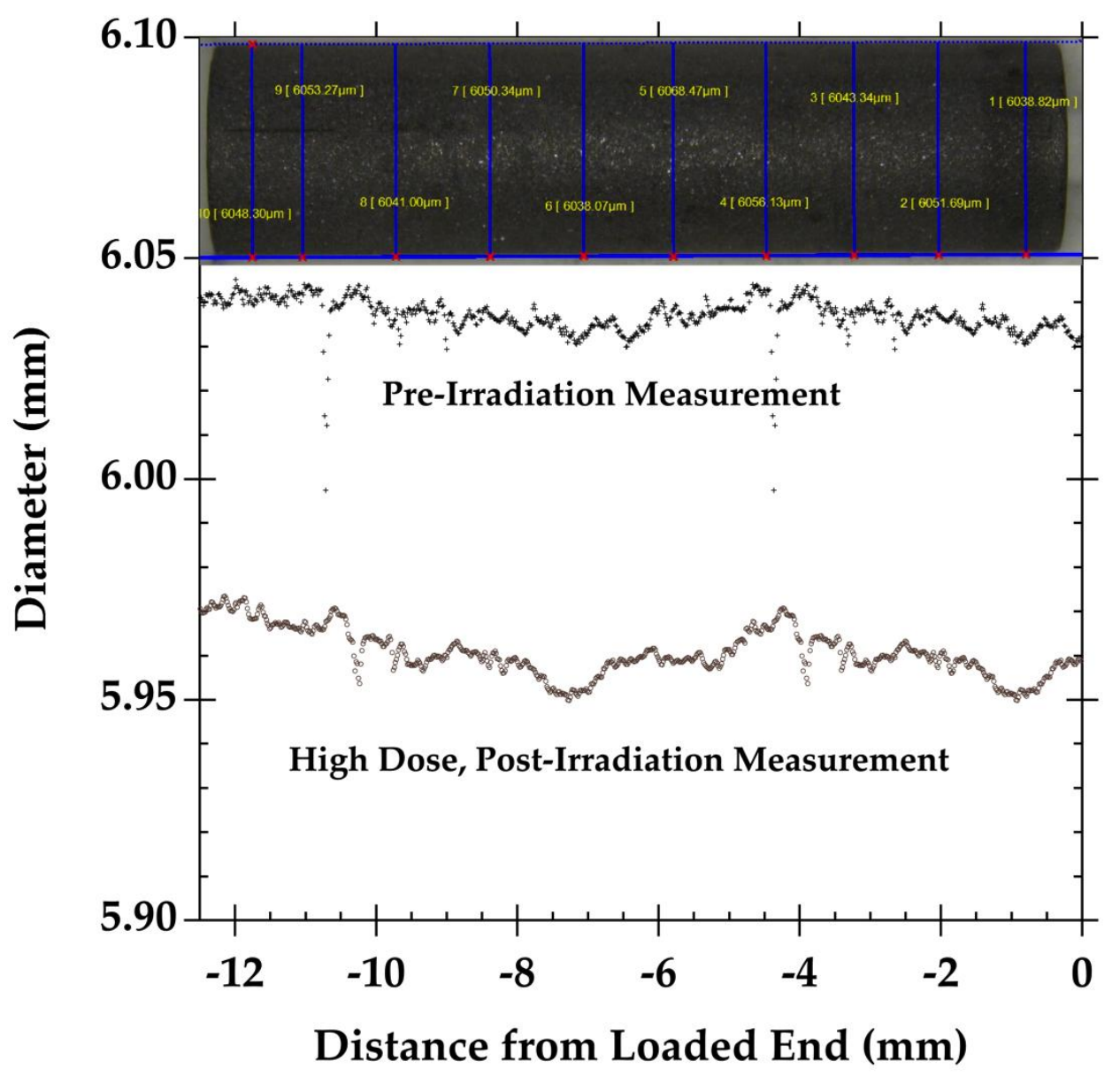


Figure 6: Pre and post irradiation laser profilometry scan from the loaded end of the R11A7 (highest fluence) loaded and free graphite sample. Inset (top) shows the optical image of the sample. Note: laser scan is limited to half the length of the sample.

Graphite irradiation creep is commonly described as the combination of primary and steady state components as follows:

$\varepsilon_{\mathrm{C}}=a\left(\sigma / \mathrm{E}_{\mathrm{o}}\right)\{1-\exp (-b \gamma)\}+\mathrm{K} \sigma \gamma$

Where $\varepsilon_{\mathrm{c}}$ is the irradiation-induced creep strain, $a$ and $b$ are fitting parameters for the primary creep, $\gamma$ is the irradiation fluence, $\sigma$ is the applied load, $E_{o}$ the initial elastic modulus, and $\mathrm{K}$ is the (steady state) creep coefficient. Assuming the fluences of this study are great enough such that the primary creep component can be ignored, the steady state creep coefficient $\mathrm{K}$ is found simply as follows:

$\mathrm{K}=\varepsilon_{\mathrm{C}} / \sigma \gamma(2)$

Oku [15] [16] [17] discussed tensile creep of a near isotropic graphite (including IG110) at approximately $900^{\circ} \mathrm{C}$ for an irradiation fluence in the range of 0.5 to $1.1 \times 10^{25}$ $\mathrm{n} / \mathrm{m}^{2}$ (E> $\left.29 \mathrm{fj}\right)$. Included in his work is an analysis of historic data irradiated over a wide temperature range, suggesting a monotonic increase in the irradiation creep coefficient, $\mathrm{K}$, with irradiation temperature. Over the $\sim 200^{\circ} \mathrm{C}$ to $\sim 1300^{\circ} \mathrm{C}$ range of historic data discussed, the steady state creep coefficient increases from a range of 1-3 to $>5$ (x 10$\left.{ }^{29}\left(\mathrm{MPa} / \mathrm{m}^{2}\right)^{-1}\right)$, respectively. Similarly, Kunimoto [18] and Shibata [19] have provided an analysis predicting the behavior of IG-110 based on limited irradiation data and suggested that the steady state creep coefficient also increases from a value of $\sim 1.2$ to 2.4 $\left(10^{-29}\left(\mathrm{MPa} / \mathrm{m}^{2}\right)^{-1}\right)$ as the irradiation temperature increases from 400 to $1000^{\circ} \mathrm{C}$.

For the samples in this experiment the creep strain is approximated as the difference between the loaded and unloaded trend lines of Figure 4. The dimensional change utilized for determining creep in the present work is measured at room temperature. It is noted that the convention in Europe and the United Kingdom is to determine the length difference at the irradiation temperature. However, for the relatively low irradiation temperature of this work the difference in convention is not significant.

Using the bellows load measured at a post-irradiation temperature of $400^{\circ} \mathrm{C}$ the irradiation creep compliance was found using equation (2) to be $\sim 1.73\left(\mathrm{x} 10^{-29}\left(\mathrm{MPa} / \mathrm{m}^{2}\right)^{-}\right.$ $\left.{ }^{1}\right)$. These values of creep coefficients are consistent with the approximation provided by the previous researchers and within the trend provided by Oku [17], though it would appear that the empirical correlation developed by Shibata $(\mathrm{K}=0.7163 \exp (0.0012 \mathrm{~T})$, where $\mathrm{T}$ is in Celsius) undervalues the creep coefficient at low temperature. 


\subsection{Thermal Conductivity and Coefficient of Thermal Expansion}

For the irradiation temperatures of this study the currently available data on thermal conductivity degradation of IG-110 or similar isotropic graphite is limited. Maruyama [13] reports on IG-110 irradiated to a fluence of less than $0.1 \times 10^{25} \mathrm{n} / \mathrm{m}^{2}$ at $200^{\circ} \mathrm{C}$ while Ishiyama [14] provides data on material irradiated at $600^{\circ} \mathrm{C}$ to $>10^{26} \mathrm{n} / \mathrm{m}^{2}$. A more relevant study is found in the JAEA report by Shibata [19] In the Shibata report thermal conductivity is provided in the range from 400 to $1200^{\circ} \mathrm{C}$ and over a fluence range spanning the current study. The results of his analysis suggest that IG-110, for similar temperature to the current work, should have a saturated thermal conductivity by approximately $0.5 \times 10^{25} \mathrm{n} / \mathrm{m}^{2}(\mathrm{E}>0.1 \mathrm{MeV})$ at a value of $\sim 19 \mathrm{~W} / \mathrm{m}-\mathrm{K}$. Figure 7 provides the measured thermal diffusivity and calculated thermal conductivity for the lowest fluence irradiation of the current study $\left(2.4 \times 10^{25} \mathrm{n} / \mathrm{m}^{2}\right)$, about a factor of five times the assumed saturation fluence of Shibata and Kunimoto.

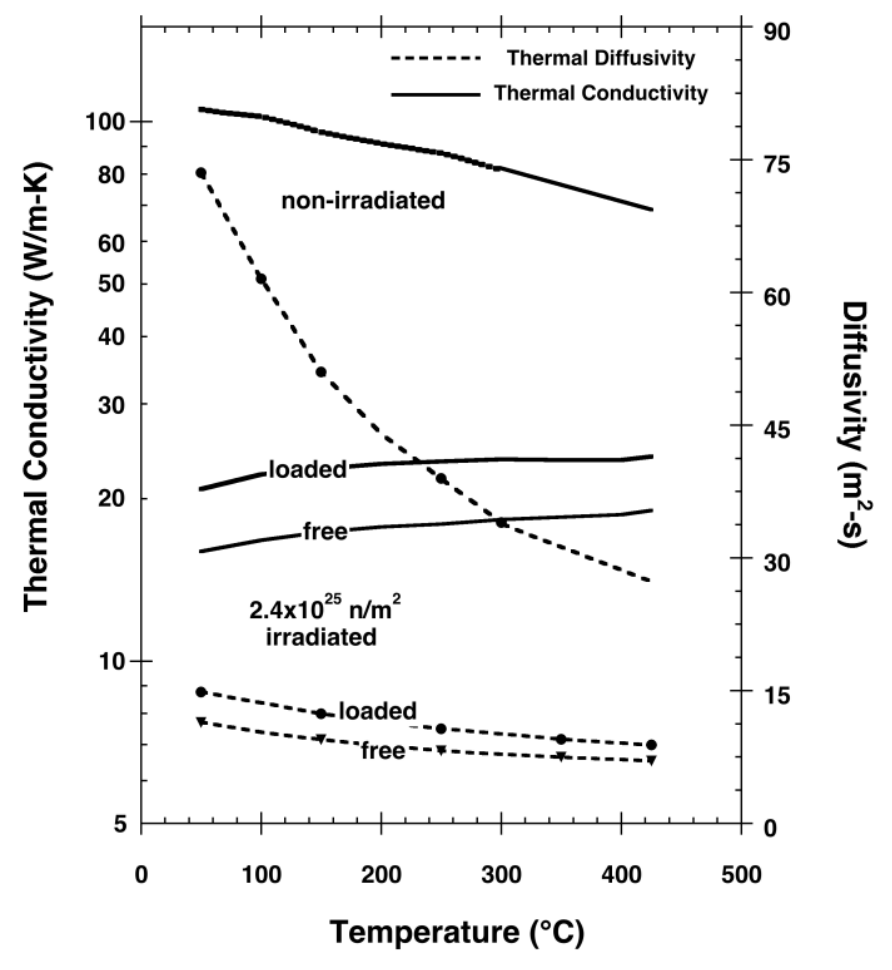

Figure 7: Calculated thermal conductivity and measured thermal diffusivity of loaded and free IG-110 irradiated to at the lowest fluence of this experiment $\left(2.4 \times 10^{25} \mathrm{n} / \mathrm{m}^{2} \mathrm{E}>0.1\right.$ $\mathrm{MeV}$.) Conductivity measured in the WG direction.

As seen in the figure, a slight difference between the loaded and free sample is observed, with the loaded sample possessing higher thermal conductivity. Moreover, for this lowest fluence specimen a temperature dependence of thermal conductivity (driven by umklapp scattering) still exists. In the upper fluence range of this study (4.6 to $9.3 \times 10^{25}$ $\mathrm{n} / \mathrm{m}^{2} \mathrm{E}>0.1 \mathrm{MeV}$ ) the thermal conductivity saturates at approximately $15 \mathrm{~W} / \mathrm{m}-\mathrm{K}$ 
measured at $50^{\circ} \mathrm{C}$, or $19 \mathrm{~W} / \mathrm{m}-\mathrm{K}$ at the irradiation temperature of $\sim 400^{\circ} \mathrm{C}$. The increasing thermal conductivity as measured from $50^{\circ} \mathrm{C}$ to $400^{\circ} \mathrm{C}$ (Figure 7) is primarily due to the increased specific heat over this range. The normalized saturation value is therefore consistent with the Shibata analysis, though saturation occurs at a much higher fluence level. The present data is also consistent with the single data point of Maruyama, who reported a room temperature thermal conductivity value of $26.9 \mathrm{~W} / \mathrm{m}-\mathrm{K}$ at a fluence approximately half the lowest fluence of the present study, confirming the trend towards saturation indicated by the present data. It is noted that one must take the usual caution regarding temperature and fluence uncertainties when comparing unrelated data-sets.

While defect scattering has near completely dominated the thermal conductivity for the samples in this irradiation, a measurable difference in thermal conductivity between the loaded and free sample appears to exist, with slightly higher conductivity offered by the loaded sample in the direction of loading. As seen in the figure the thermal conductivity is marginally higher (20.8 as compared to $15.9 \mathrm{~W} / \mathrm{m}-\mathrm{K})$ in the loaded direction for this lowest irradiation fluence. The two higher fluence capsules also had a similar, albeit lesser, difference in thermal conductivity between loaded and free samples. Thermal conductivity for the free and loaded samples of the highest fluence capsule exhibited the same trend, though as no information was available on the true temperature of the R11A8 (free) capsule any comparison is unwise. As will be discussed in a later section the influence of compressive loading on the sample is to reduce the overall pore volume of the sample. As would be expected, by reducing the overall porosity of the graphite the relative thermal conductivity would be expected to increase, which has occurred for the loaded samples. Alternative explanations, including reorientation of the internal structure of the graphite may also be a contributor to the thermal transport.

The thermal expansion behavior in the WG direction for loaded and free IG-110 is provided in Figure 8, compared to non-irradiated specimens for the lowest fluence condition $\left(2.2 \times 10^{25} \mathrm{n} / \mathrm{m}^{2}, \mathrm{E}>0.1 \mathrm{MeV}\right)$ on the left of the figure and the higher fluence intervals on the right of the figure. A clear increase in CTE occurs following the lowest fluence irradiation with a greater increase for the loaded specimen. For the three higher fluence capsules (rightmost of fig 8), the CTE change increase has apparently gone through a maximum, as seen in Figure 9, with the CTE of the highest-fluence sample somewhat less than the second-highest fluence. It is noted that the sample-to-sample variation in CTE for IG-110 is extraordinarily low. This combined with a well-behaved, repeatable measurement system provides error bars associated with CTE for figures 8 and 9 that are negligibly small. Neither the increased CTE with irradiation damage or the relative maximum prior to $1 \times 10^{26} \mathrm{n} / \mathrm{m}^{2} \mathrm{E}>0.1 \mathrm{MeV}$ for the non-crept free samples is surprising and is predicted by the analysis of Kunimoto [18] and Shibata [19] . The magnitude of CTE change (for the non-crept, free sample) is consistent with the analysis of Kunimoto [18] , though about a factor of two higher than suggested by Shibata [19]. 

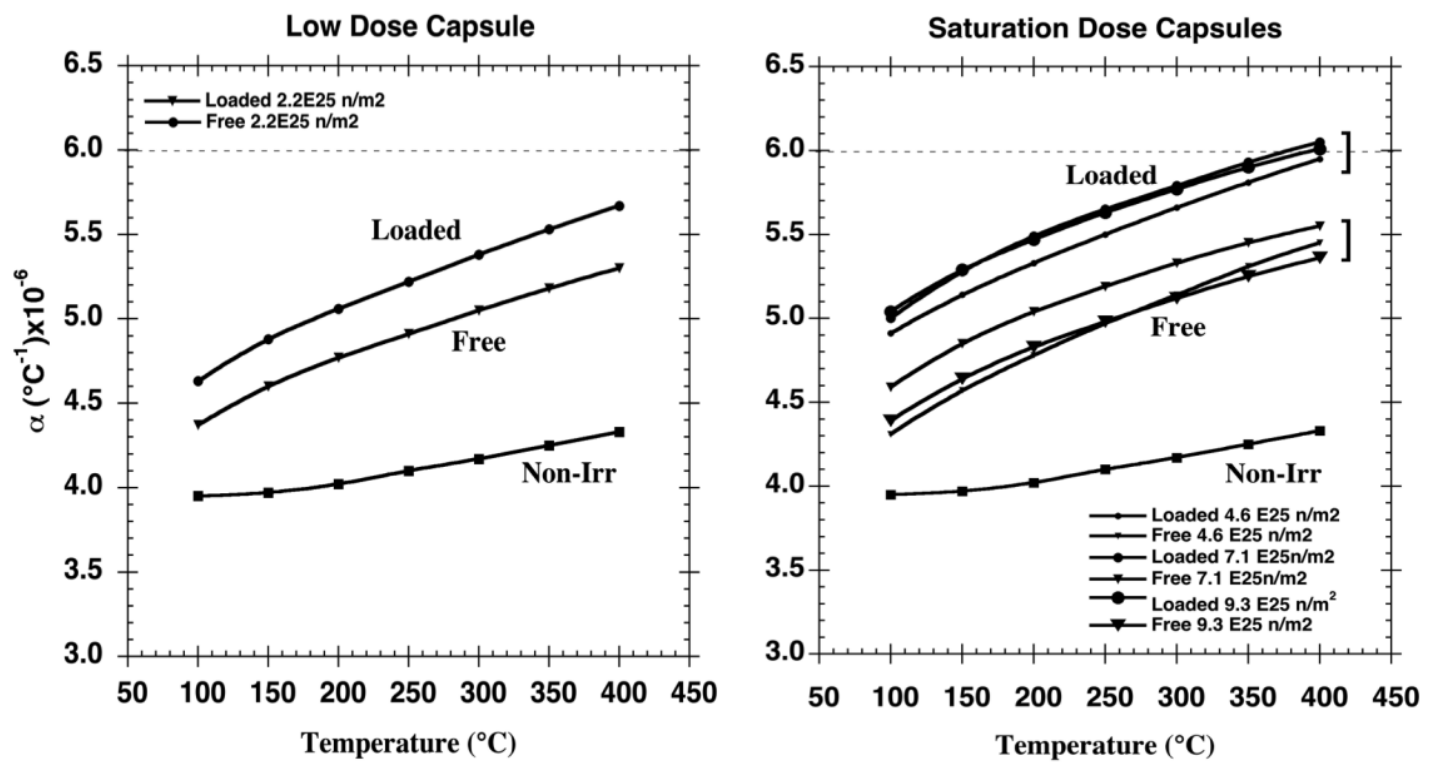

Figure 8: Coefficient of thermal expansion (average from $20^{\circ} \mathrm{C}$ to measurement temperature) for non-irradiated and irradiated specimens. Left figure provides the loaded and free specimens for the low fluence capsule $\left(2.2 \times 10^{25} \mathrm{n} / \mathrm{m}^{2}, \mathrm{E}>0.1 \mathrm{MeV}\right)$ while the right figure provides data for the three higher fluence capsules. CTE measured in the WG direction. 


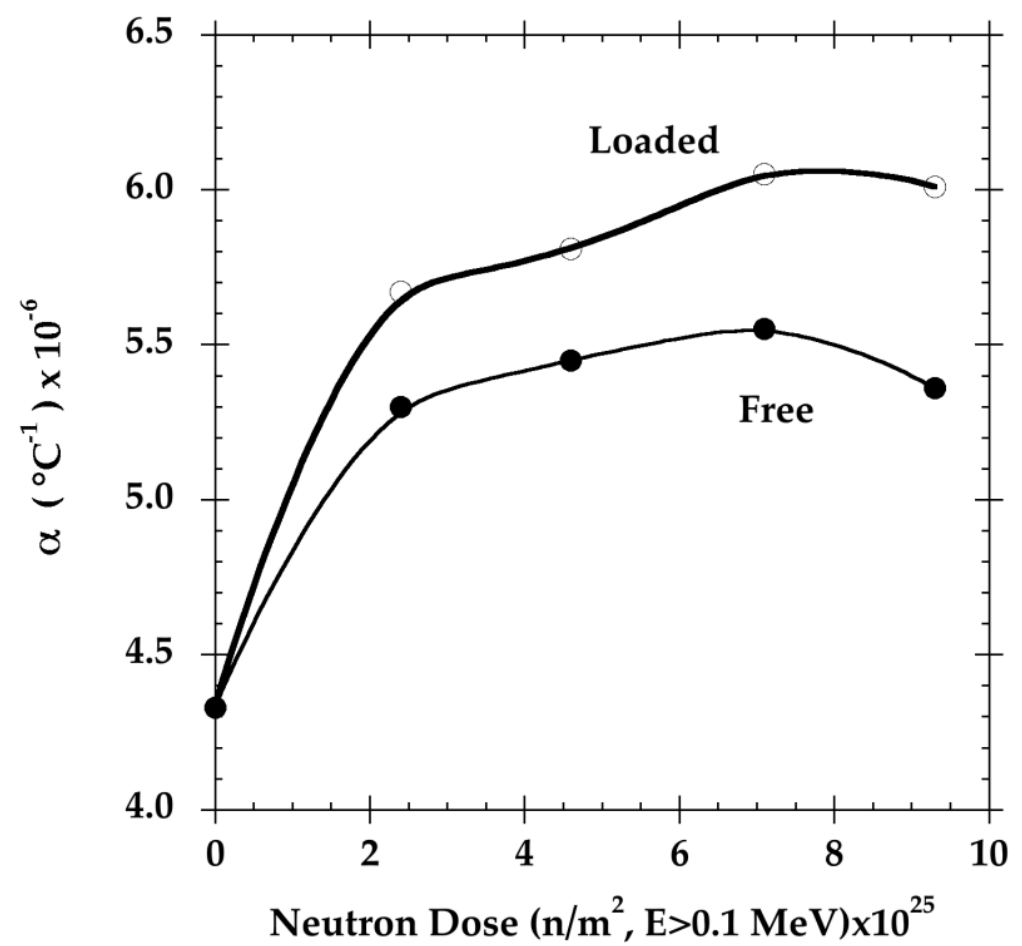

Figure 9 Coefficient of thermal expansion at $400^{\circ} \mathrm{C}$ (averaged from $20-400^{\circ} \mathrm{C}$ ) for loaded and free IG-110 as a function of neutron fluence. CTE measured in the WG direction.

\subsection{Elastic Modulus}

The increased dynamic elastic modulus at room temperature for the samples is given in Figure 10 showing a rapid increase in modulus and an apparent transition to near saturation for the highest fluence of this study, consistent with the behavior predicted by Kunimoto for $400^{\circ} \mathrm{C}$ irradiation. [18] Unfortunately, the dynamic modulus was not measured for each sample prior to irradiation and given the variability from sample to sample (reflected in the \pm 1 sigma error bar provided of $\sim 1 \mathrm{GPa}$ for this dynamic modulus measurement) it is difficult to speculate on the effect of loading. However, a trend towards lower modulus for the loaded specimens may be inferred. 


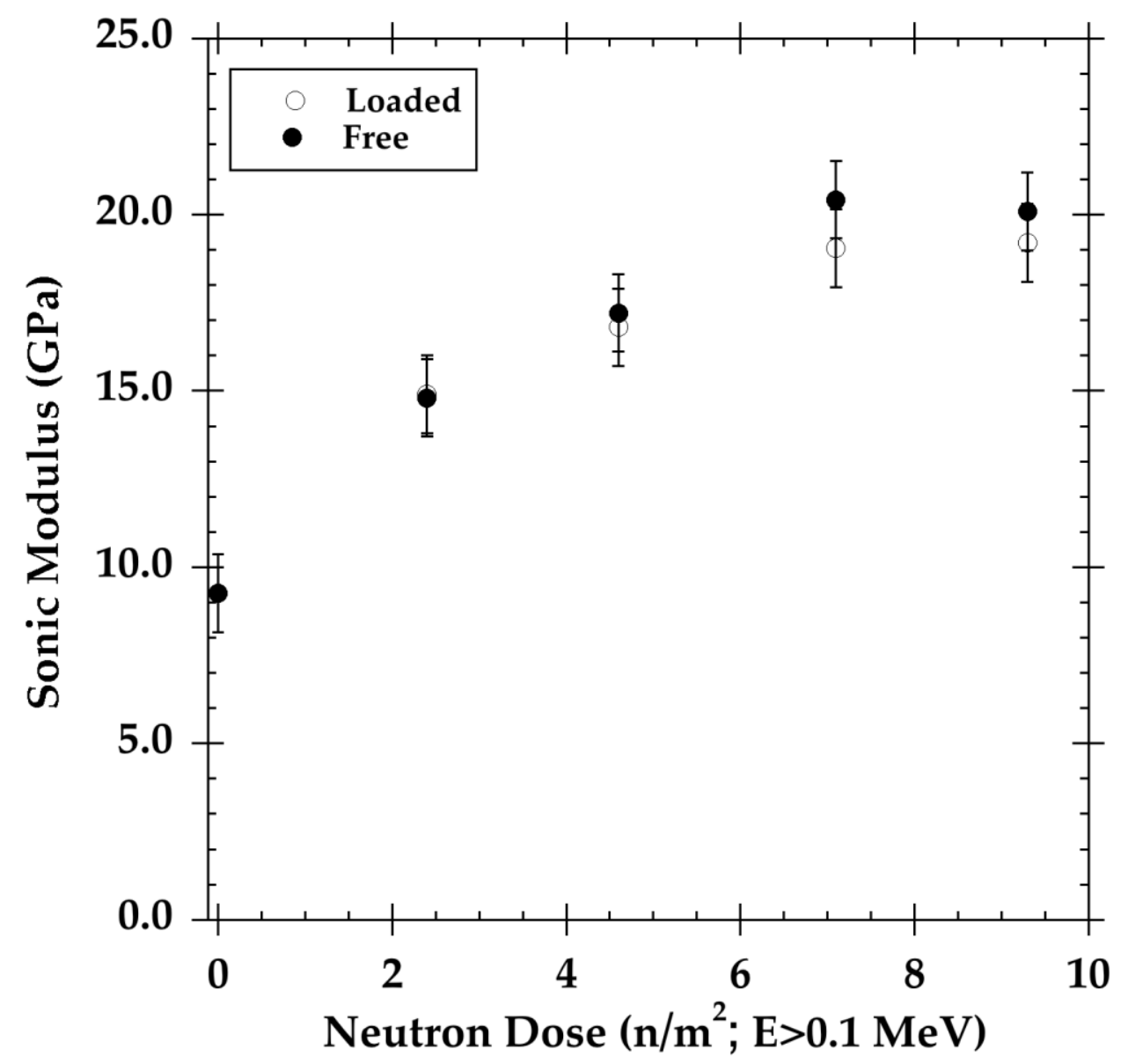

Figure 10: Effect of neutron irradiation on dynamic elastic modulus of IG-110.

\subsection{Open Porosity Evolution}

Nitrogen adsorption at $77 \mathrm{~K}$ provides information on open porosity in graphite in the size range from 0.3 to about $40 \mathrm{~nm}$, and complements the information available from mercury porosimetry which accesses the pores with sizes from $100 \mu \mathrm{m}$ to about $5 \mathrm{~nm}$. The specific surface area (SSA) calculated by the Brunauer - Emmett - Teller (BET) method is a standard measure of total internal surface of accessible pores. The total volume of open pores was estimated from the total amount of nitrogen condensed up to the highest pressure of the measurements, usually $99 \%$ of the nitrogen saturation vapor pressure at its boiling temperature. An approximate value of the average size of open pores can be estimated from the surface and volume information. With the additional assumption that the prevalent shape of open pores is near cylindrical, the average pores diameter $\mathrm{d}(\mathrm{nm})$ is obtained as $\mathrm{d}=4 \times 10^{4} \times(\mathrm{V} / \mathrm{S})$, where $\mathrm{V}\left(\mathrm{cm}^{3} / \mathrm{g}\right)$ is the total pore volume and $\mathrm{S}\left(\mathrm{m}^{2} / \mathrm{g}\right)$ is the SSA. A summary of results showing the effect of irradiation and external loading on internal porosity is presented in Figure 11.

Figure 11 shows that all specimens irradiated with no load exhibit higher SSA values than the non-irradiated sample. In addition, the SSA values of specimens irradiated under load are lower than the SSA values of their paired samples irradiated with no load. In 
particular, the SSA of graphite irradiated with no load applied first increases with fluence and then decreases, but remains higher than non-irradiated material. The total pore volume has a similar increase, whereas the average pore size mirrors the variation of the latter. This behavior suggests that irradiation initially causes a gradual shrinkage in average pore size along with the multiplication of the pores accessible to nitrogen, leading to the increase of total pore volume and of SSA. At higher fluences, this effect is partially reversed.
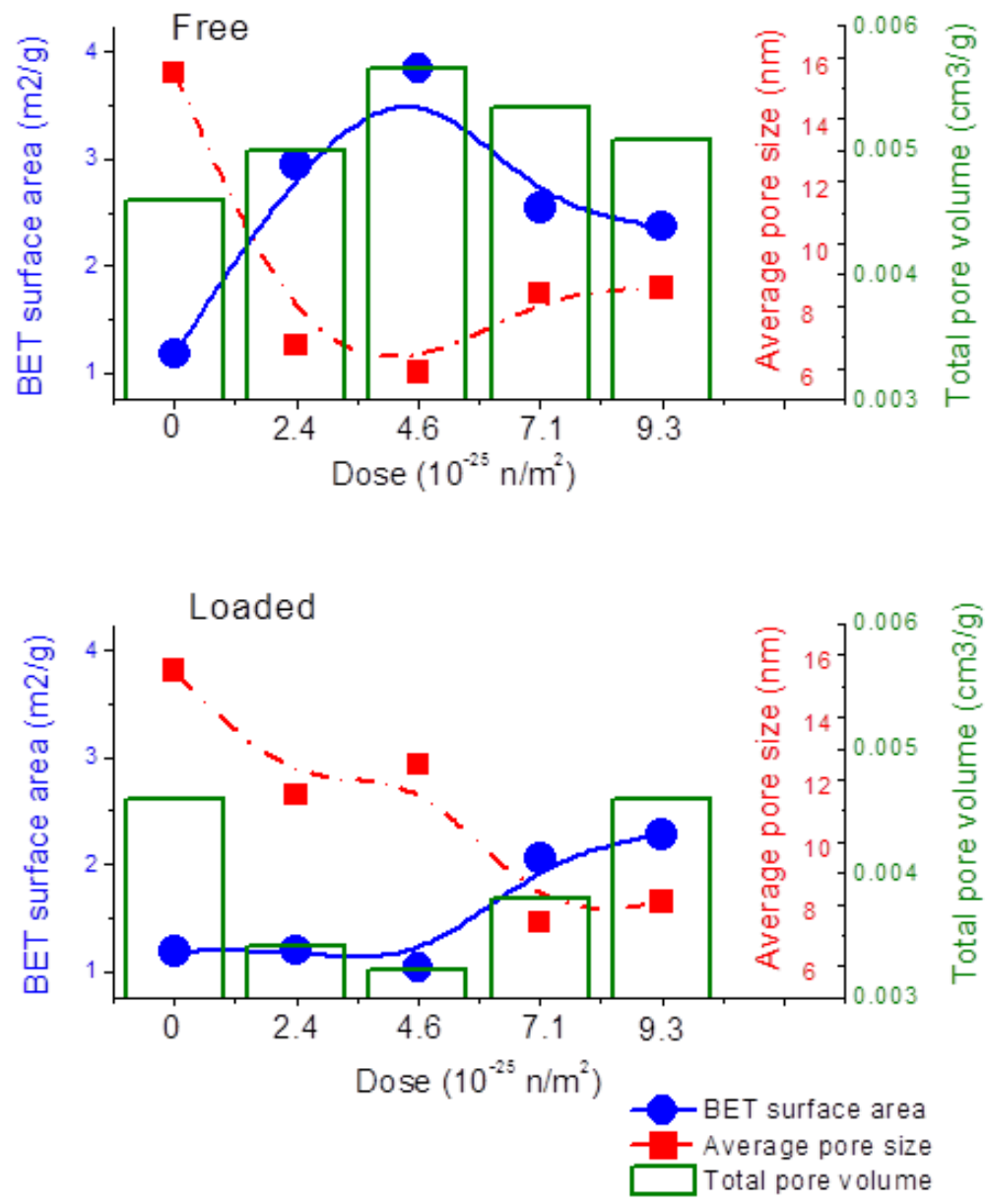

Figure 11: BET specific surface area of irradiated graphite.

For the loaded sample the specific area, rather than increasing at low fluence as observed with the free samples, diminishes. This trend is paralleled by a two-step decrease of average pore size. It appears that irradiation at low fluence under external loading causes preferential closure of larger pores, while narrow pores are affected less. For the higher fluences of this study the specific surface area and the total pore volume increase, moving towards levels closer to their non-irradiated values. Over the entire range of fluence studied a decrease in average pore size occurs, indicating that more narrow pores have 
become accessible to gas adsorption or that an absolute change in the pore size has occurred. The difference in total pore volume change between the loaded and free samples is a small fraction of the difference in volumetric change that can be obtained by Figures 4 and 5. From these results it can be concluded that any evolution in the nitrogen accessible pore structure in the $2-50 \mathrm{~nm}$ range can at most have only a small contribution towards bulk dimension and density changes reported above. A more detailed analysis revealing details on the energetic heterogeneity of internal surfaces, and the pore size distribution of open micropores $(<2 \mathrm{~nm})$ and mesopores $(2-50 \mathrm{~nm})$ is presented in a companion paper. Interestingly, that analysis suggests that the increase in total pore volume after irradiation is accompanied by multiplication of narrow mesopores, and that their internal surfaces are more energetically homogeneous than in the original, nonirradiated material.

\section{Concluding Remarks}

A new bellows-loaded capsule design has been used to provide in-situ loading of nuclear graphite for the study of physical property and microstructural evolution under neutron irradiation. Irradiation-creep for IG-110 graphite is inferred from the length changes in matched compression-loaded and non-loaded samples yielding a creep compliance of $\sim 1.73\left(10^{-29}\left(\mathrm{MPa} / \mathrm{m}^{2}\right)^{-1}\right)$ which is in general agreement with earlier, empirical relationships. Similarly, an effect of loading on thermal conductivity is measured. For the loaded graphite, which had a somewhat lower pore size and volume, the thermal conductivity was measurably higher. A significant, stress dependent increase in the coefficient of thermal expansion has been measured. While a similarly significant increase in dynamic modulus occurs due to the irradiation, no differentiation between materials irradiated with and without compressive stress was observed within experimental error. Nitrogen adsorption analysis suggests a difference in pore evolution in the $2-50 \mathrm{~nm}$ range for graphite irradiated with and without compressive stress, but this pore evolution is at most a small contributor to the overall dimensional change observed. 
Acknowledgements

The authors would like to than Joel McDuffee and Bob Sitterson for their efforts in the design and construction of irradiation capsules. Irradiations were carried out in the High Flux Isotope Reactor, an user facility funded by the Office of Science, US Department of Energy. The work presented in this manuscript was supported in part by the Advanced Fuels Campaign of the Fuel Cycle R\&D program in the Office of Nuclear Energy, and the Office of Fusion Energy Sciences US Department of Energy. The authors would like to than Mr. Mark Davies for assistance with the manuscript.

\section{References}

[1] B. T. Kelly, Physics of Graphite; Applied Science Publishers: London, 1981.

[2] X. Fang et. al., Nuclear Engineering and Design, 2012, (2012) 19.

[3] J. Kane et. al., Journal of Nuclear Materials, 415, (2011) 189.

[4] C. Contescu et. al., Carbon, 50, (2012) 3354.

[5] T. D. Burchell et. al., AGC-1 Sister Specimen Testing Data Report. ORNL/TM2009/025 (Oak Ridge, TN, May 2009). (2009).

[6] W. Jiang et. al., Thermochimica Acta, 351, (2000) 85.

[7] J. A. Vreeling, 0. Wouters, J. G. Van der Laan, Journal of Nuclear Materials, 381, (2008) 68.

[8] T. S. Byun et. al., Journal of Nuclear Materials, 439, (2013) 108.

[9] L. L. Snead, A. M. Williams, A. L. Qualls. "Revisiting the use of SiC as a Post Irradiation Temperature Monitor. ASTM STP 1447.M. L. Grossbeck, ed. ASTM International, West Conshohocken, PA, 2003."; The Effects of Radiation on Materials: 21st International Symposium, 2003.

[10] J. L. Rempe et. al., IEEE Transactions on Nuclear Science, 57, (2010) 1589.

[11] A. A. Campbell et. al., Nuclear Instruments and Methods in Physics Research

Section B: Beam Interactions with Materials and Atoms, 370, (2016) 49.

[12] S. Brunauer, P. H. Emmett, E. Teller, Journal of the American Ceram. Soc., 60, (1938) 309.

[13] T. Maruyama, M. Harayama, J. Nucl. Mat., 195, (1992) 44.

[14] S. Ishiyama et. al., J Nucl Mat, 230, (1996) 1.

[15] T. Oku, K. Fujisaki, M. Eto, J Nucl Mat, 152, (1988) 225.

[16] T. Oku, S. Ishiyama, Journal of Nuclear Science and Technology, 24, (1987) 670.

[17] T. Oku, M. Eto, S. Ishiyama, J Nucl Mat, 1990, (1990) 77.

[18] E. Kunimoto et. al. "Expansion of Irradiation Data by Interpolation and Extrapolation for Design of Graphite Components in High Temperature Gas Cooled Reactor-Evaluation of IG-110 Graphite Irradiation Data for Component Design. JAEA-Research 2009-008," 2009.

[19] T. Shibata et. al. "Draft of Standard for Graphite Core Components in High Temperature Gas-cooled Reactor. JAEA-Research 2009-042.,” 2009. 